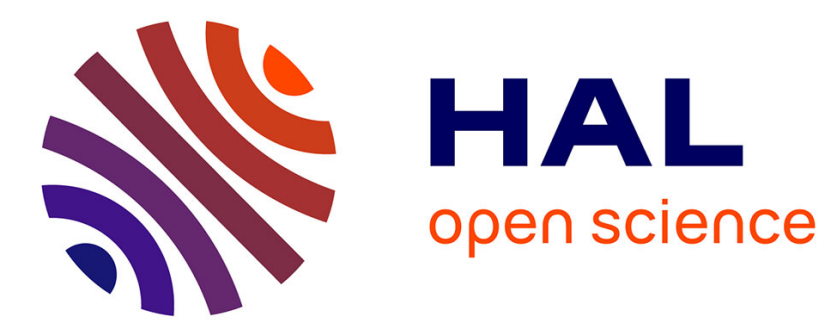

\title{
Negative density-dependent dispersal in tsetse flies: A risk for control campaigns?
}

Thierry de Meeûs, Sophie Ravel, Philippe Solano, Jérémy Bouyer

\section{To cite this version:}

Thierry de Meeûs, Sophie Ravel, Philippe Solano, Jérémy Bouyer. Negative density-dependent dispersal in tsetse flies: A risk for control campaigns?. Trends in Parasitology, 2019, 35 (8), pp.615-621. 10.1016/j.pt.2019.05.007 . hal-02624319

\section{HAL Id: hal-02624319 \\ https://hal.inrae.fr/hal-02624319}

Submitted on 25 Oct 2021

HAL is a multi-disciplinary open access archive for the deposit and dissemination of scientific research documents, whether they are published or not. The documents may come from teaching and research institutions in France or abroad, or from public or private research centers.
L'archive ouverte pluridisciplinaire HAL, est destinée au dépôt et à la diffusion de documents scientifiques de niveau recherche, publiés ou non, émanant des établissements d'enseignement et de recherche français ou étrangers, des laboratoires publics ou privés.

\section{다)(1) $(5$}

Distributed under a Creative Commons Attribution - NonCommerciall 4.0 International 


\section{Negative density dependent dispersal in tsetse flies: a risk for control campaigns?} Thierry De Meeûs ${ }^{1 *}$, Sophie Ravel ${ }^{1}$, Philippe Solano ${ }^{1}$ and Jérémy Bouyer ${ }^{1,2,3}$

$4 \quad{ }^{1}$ Intertryp, IRD, Cirad, Univ Montpellier, Montpellier, France.

$5 \quad{ }^{2}$ Astre, Cirad, Inra, Montpellier, France.

$6{ }^{3}$ Insect Pest Control Laboratory, Joint Food and Agriculture Organization of the United Nations/International Atomic Energy Agency Program of Nuclear Techniques in Food and Agriculture, A-1400 Vienna, Austria

9 *Corresponding author: thierry.demeeus@ird.fr

11 Keywords: Glossina, Sleeping sickness, Nagana, Control campaigns, Effective population size, Migration rate.

14 Abstract

Tsetse flies are vectors of parasites that cause diseases responsible for significant economic losses and health issues in sub-Saharan Africa, including sleeping sickness in

17 humans and nagana in domestic animals. Efficient vector control campaigns require good

18 knowledge of the demographic parameters of the targeted populations. In the last decade, 19 population genetics emerged as a convenient way to measure population densities and

20 dispersal in tsetse flies. Here by revealing a strong negative density-dependent dispersal

21 in two dimensions, we suggest that control campaigns might unleash dispersal from

22 untreated areas. If confirmed by direct measurement of dispersal before and after control 23 campaigns, area wide and/or sequential treatments of neighboring sites will be necessary 24 to prevent this issue. 


\section{Tsetse fly control and population genetics tools}

Flies of the genus Glossina are the vectors of trypanosomes, parasites responsible for diseases that lead to a significant disease burden and economic losses in sub-Saharan Africa. In humans, tsetse flies transmit trypanosomiasis, also known as sleeping sickness" which is usually mortal if the patient is not treated [1]. In animals, particularly cattle, it causes nagana, a disease known to cause considerable economic losses in sub-Saharan Africa $[2,3]$. Vector control has become a recognized key component in the management of these plagues [3-5]. Nevertheless, to optimize control campaigns, good knowledge of the biology of the target populations is needed, in particular their density and dispersal ability [6]. Lack of such knowledge has indeed been implicated in the failure of some elimination campaigns due to the raid reinvasion of treated populations by local flies or by flies from neighboring sites [7]. Population genetics (see Glossary) offers useful tools [8] in particular for tsetse flies [3].

Considerable work has been undertaken in recent years to study the population biology of different species of tsetse flies in a range of different countries with varying success in terms of population density and dispersal estimates (e.g. see [4, 7, 9-12]).

The best way to accurately estimate dispersal of a given population is isolation by distance. Isolation by distance is a common feature of population structure in which the genetic relatedness (or genetic distance) between individuals or subpopulations (see Box 1) is a decreasing function of the geographic distance separating them (the shorter the distance between them, the more similar they are). When such genetic distances are known, an isolation by distance model can be built and its significance tested (Box 2). If it is significant and if effective population density $D_{e}$ can be estimated, then dispersal $\delta$ can be extracted from the model (Box 2).

In the last decade, several data sets have provided the opportunity for such inferences for different tsetse fly species in different countries in Africa: Glossina palpalis 
52 gambiensis along the northern reaches [13] and the southern reaches of the Mouhoun

53 River [14] in Burkina-Faso (in one dimension=1D, see Box 2); G. palpalis palpalis in

54 Cameroon (two dimensions=2D, see Box 2) [11, 15]; G. palpalis gambiensis and G.

55 tachinoides in Southern Burkina Faso (2D) across river basins [9]; G. tachinoides in Ghana

56 (2D) [7]; G. fuscipes fuscipes from Uganda [12, 16], in Tanzania and Kenya [17] (2D); G.

57 pallidipes from Kenya Nguruman escarpment and Ruma [18] and from the Serengeti Park

58 Reserve in Tanzania (2D) [10]. This offered the opportunity to check if any relationship

59 existed between these different inferences of population density and dispersal. The

60 objective of the present paper is to discuss the evidence that tsetse dispersal may be

61 density dependent.

62

\section{Regression between dispersal and effective population density}

The detailed protocol for using or reanalyzing available data and computing the necessary parameters are given in the supplementary file S1. Positive density dependence dispersal occurs when dispersal increases with density, as expected if individuals leave to escape overcrowding. Negative density dependent dispersal happens when crowded populations can no longer accept any immigrants. To investigate evidence for density-dependent dispersal in tsetse, we computed effective population densities as the ratio of effective population size $\left(N_{e}\right)$ to the average surface area occupied by a population (Box 2). For the sake of homogeneity across studies, $N_{e}$ was estimated using linkage disequilibrium methods [19-22] and the surface area occupied by a subpopulation corresponds to the surface of subsamples when available or the disc defined by the minimum distance between the closest subsamples taken as the diameter of a population (Box 2). Like for dispersal, we extracted slope estimates and 95\% bootstrap confidence intervals over loci $(95 \% \mathrm{Cl})$ (when available) to compute dispersal 
78 both geographic and genetic distances to perform the regression. We kept only data with 79 2D population structure regressions [7, 9-11]. For some studies [12, 15-18], we needed to re-compute several statistics, as explained in detail in supplementary file S1. Only two studies where 1D dispersal inferences were available along the Mouhoun River in Burkina Faso [13, 14] were not included. According to Watts et al. [23], high mutation rates have a stronger effect on the accuracy of the method in linear habitats than in two-dimensional habitats and this may affect analyses of riparian systems. The fact that the metric in 1D is in $\mathrm{m}$ and in $2 \mathrm{D}$ in $\mathrm{m}^{2}$ also makes comparisons difficult. Additionally, we wanted to check that the relationship was not due to a systematic bias in $D_{e}$ and $\delta$ estimates by using independent estimates of $D_{e}$ and census densities (see below). $N_{e}$ can strongly depend on the reproductive system, on fluctuations in population sizes and/or generation overlap. This last point should be minimized if samples taken at intervals of not more than two months are used, as we recommend (Supplementary File S1). We also expect that $N_{e}$ is strongly correlated with the census size of the population, otherwise all population genetics studies of tsetse flies would need to be called into question. Finally, a census of flies captured during the studies, when available, was also analysed and, likewise, was seen to

94 be correlated with the real census of the corresponding tsetse populations [24]. population densities $\left(D_{e}\right)$, and in $1 \mathrm{~b}$ with census population densities $\left(D_{c}\right)$. An exponential, tight and negative relationship can be seen between population density and dispersal. The regression explains most of the variance $\left(R^{2} \approx 0.85\right.$ for $D_{e} ; R^{2} \approx 0.86$ for $\left.D_{c}\right)$. Spearman's rank correlation testing with $\mathrm{Rcmdr}$ package $[25,26]$ for $\mathrm{R}[27]$ gave significant $p$-values (0.0056 and 0.0167 for $D_{e}$ and $D_{c}$ respectively). 
density dependence (the intuitively expected sign of correlation), in particular in insects

$105[28,29]$. "The occurrence of negative density dependent dispersal is in agreement with the "social fence" and related hypotheses which have been proposed in particular for small mammal populations, where movements are increasingly inhibited by aggression at high densities" [30]. Negative density dependence can result from Allee effects or because other factors, such as predation, interact with density to negatively influence dispersal [28].

110 In the case of insects, if there is a trade-off between wing development and reproductive 111 capacity, dispersal may also be promoted by harsh local conditions, which can be 112 correlated with low levels of population density, especially when dispersal is costly [31]. There are nevertheless several reported cases of negative density dependent

114 dispersal. Experimental studies on insects have shown that juvenile hormone titer, which is 115 influenced by the diet at earlier stages, can influence wing size and hence dispersal capacities [31]. Negative density dependence has also been reported in the northern pine processionary moth Thaumetopoea pinivora [29].

\section{Dispersal is strongly density dependent in tsetse flies}

Negative density dependent dispersal in tsetse populations has been known for some time (Box 3) but its intensity has not been measured to date. The exponential negative density dependence of dispersal observed in the present study raises several questions. The phenomenon implies that competition for space is very harsh and probably occurs mainly during feeding on the host (Box 3). When densities are high, all the sites are crowded and both hosts and local tsetse are accustomed to each other (Box 3). Naïve immigrants from remote sites are characterized by low feeding success, thus increasing

127 their mortality. When population densities are low, densities may vary in both space and 128 time, which renders immigration much easier and allows long-range immigration. In an 
129 empty spot with no other tsetse competitors and naïve hosts, even exhausted immigrants

130 from remote sites can safely settle. This pattern could also reflect favorable host densities.

131 When densities of favorable hosts are low, tsetse flies need to keep moving to find a

132 suitable blood meal.

133 Since control campaigns aim at considerably reducing tsetse densities, they may

134 unleash dispersal and relatively rapid reinvasion of treated zones from neighboring or even

135 remote sites, as may have occurred in Ghana [7] and certainly occurred in Ivory Coast

136 [32]. Reinvasion of flies in areas depleted as a result of vector control has been well

137 documented for quite some time and emphasizes the role played by fly movement in the

138 development of control strategies [33]. Some evidence suggests strong density-

139 dependence associated with this phenomenon: the bigger the decrease in the population,

140 the higher the potential for reinvasion in a continuous tsetse belt [33]. These authors also

141 report that Hargrove's models use density-dependent mortality, but not dispersal [34]. This

142 limitation has already been reported and actually concerns all existing models [35].

143 Density-dependent dispersal was only recently incorporated in a tsetse population

144 dynamics model, based on a sigmoidal density-dependent dispersal rate adapted for

145 individuals competing for access to resources [36]. Such immigrations are dangerous for

146 two reasons: i) because they can severely jeopardize the sustained success of control

147 campaigns; ii) these new immigrants can bring with them pathogenic agents or more

148 virulent strains that were not present before the campaign and were unable to invade the

149 zone because of competition for space; and iii) these immigrants may replace the local

150 population and adapt to local pathogens differently and exhibit new vectorial capacities

151 compared to the former population. What is more, negative density-dependent dispersal

152 may partially explain some observed failures. As underlined by Rogers and Randolph [32]:

153 "Control programmes must recognize that the efficacy of population suppression may well

154 be reduced at the low levels of density that need to be maintained in order to reduce the 
rates of disease transmission, because at these low levels, tsetse may show entirely unexpected demographic vigor, due to the absence of normal density dependent constraints". If suitable host density is the only force driving the observed pattern, then control campaigns would not be expected to have any impact on dispersal.

\section{Concluding Remarks}

More data on isolation by distance slopes and effective population density estimates are needed to confirm the exact relationship found so far. One-dimensional isolation (along river courses) is particularly rare up to now. Most species we studied belong to the palpalis subgenus and one species (G. pallidipes) to the morsitans subgenus. The trends highlighted here probably apply to all tsetse flies but more studies are needed to generalize the trends we found to the whole genus. Studies should also focus on areas where tsetse control has been implemented. Considering that our results strongly support negative density-dependent dispersal, it will be crucial to evaluate the strength of the phenomenon using direct methods like mark-release-recapture in the field before and after control, for instance, combined with population genetics analyses. Alternatively, the prediction that neighboring flies will recolonize the treated area after the campaign ends can easily be assessed with sampling and genotyping before and after a control campaign in the treated area and its surroundings. If confirmed, and if one wants to avoid reinvasions and their associated issues (see Outstanding Questions), it will be even more important to find a way to isolate zones after treatment [4] and to sequentially treat neighboring areas harboring tsetse flies. Finally, several published results could not be included in the present review because of data unavailability. Data availability is now mandatory in several journals but we think this should be generalized. 
180 Acknowledgements

181 We thank Winnie Okeyo, Norah Saarman, Oliver Manangwa, Trésor Tito Tanekou

182 Melachio, Adalgisa Caccone and Chaz Hyseni for providing assistance with their data 183 sets, which meant we could compute parameters that were missing in their original papers

184 and considerably enriched our perception of density dependence in tsetse flies in the 185 present review. We also thank Dr Raphaël Leblois for his advice concerning theoretical 186 issues in isolation by distance.

187 


\section{References}

189 1. Jamonneau, V. et al. (2012) Untreated human infections by Trypanosoma brucei 190 gambiense are not 100\% Fatal. PLoS Negl Trop Dis 6 (6), e1691.

191 2. Van den Bossche, P. et al. (2010) A changing environment and the epidemiology of 192 tsetse-transmitted livestock trypanosomiasis. Trends Parasitol 26 (5), 236-243.

193 3. Solano, P. et al. (2010) How can tsetse population genetics contribute to African 194 trypanosomiasis control? Trends Parasitol 26 (5), 255-263.

195 4. Bouyer, J. et al. (2015) Mapping landscape friction to locate isolated tsetse populations 196 candidate for elimination. Proc Natl Acad Sci U S A 112 (47), 14575-14580.

197 5. Diall, O. et al. (2017) Developing a progressive control pathway for African animal 198 trypanosomosis. Trends Parasitol 33 (7), 499-509.

199 6. Vreysen, M.J.B. et al. (2013) Tsetse flies: Their biology and control using area-wide 200 integrated pest management approaches. J Invertebr Pathol 112, S15-S25.

201 7. Adam, Y. et al. (2014) Genetic comparison of Glossina tachinoides populations in three 202 river basins of the upper west region of Ghana and implications for tsetse control. Infect 203 Genet Evol 28, 588-595.

204 8. De Meeûs, T. et al. (2007) Population genetics and molecular epidemiology or how to 205 "débusquer la bête". Infect Genet Evol 7 (2), 308-332.

206 9. Kone, N. et al. (2011) Contrasting population structures of two vectors of African 207 trypanosomoses in Burkina Faso: consequences for control. PLoS Negl Trop Dis 5 (6), 208 e1217.

209 10. Manangwa, O. et al. (2019) Detecting Wahlund effects together with amplification 210 problems: cryptic species, null alleles and short allele dominance in Glossina pallidipes 211 populations from Tanzania. Mol Ecol Res 19, 757-772.

212 11. Mélachio, T., Tito, Tanekou et al. (2011) Population genetics of Glossina palpalis 213 palpalis from central African sleeping sickness foci. Parasites and Vectors 4, 140. 
214 12. Opiro, R. et al. (2017) Genetic diversity and population structure of the tsetse fly

215 Glossina fuscipes fuscipes (Diptera: Glossinidae) in Northern Uganda: Implications for 216 vector control. PLoS Negl Trop Dis 11 (4), e0005485.

217 13. Bouyer, J. et al. (2009) Population sizes and dispersal pattern of tsetse flies: rolling on 218 the river? Mol Ecol 18, 2787-2797.

219 14. Bouyer, J. et al. (2010) Population structure of Glossina palpalis gambiensis (Diptera:

220 Glossinidae) between river basins in Burkina-Faso: consequences for area-wide integrated 221 pest management. Infect Genet Evol 10 (321-328).

222 15. Mélachio, T.T. et al. (2015) Effect of sampling methods, effective population size and 223 migration rate estimation in Glossina palpalis palpalis from Cameroon. Infection, Genetics 224 and Evolution 33, 150-157.

225 16. Hyseni, C. et al. (2012) The population structure of Glossina fuscipes fuscipes in the 226 Lake Victoria basin in Uganda: implications for vector control. Parasit Vect 5, 1-14.

227 17. Manangwa, O. et al. (2017) Genetic diversity of Glossina fuscipes fuscipes along the 228 shores of Lake Victoria in Tanzania and Kenya: implications for management. Parasit 229 Vectors $10(1), 268$.

230 18. Okeyo, W.A. et al. (2017) Temporal genetic differentiation in Glossina pallidipes tsetse 231 fly populations in Kenya. Parasit Vect 10 (1), 471.

232 19. Bartley, D. et al. (1992) Use of linkage disequilibrium data to estimate effective size of 233 hatchery and natural fish populations. Conserv. Biol. 6 (3), 365-375.

234 20. Do, C. et al. (2014) NeEstimator v2: re-implementation of software for the estimation of 235 contemporary effective population size $\left(N_{e}\right)$ from genetic data. Mol Ecol Res 14 (1), 209236214.

237 21. Peel, D. et al. (2013) Accounting for missing data in the estimation of contemporary 238 genetic effective population size $\left(N_{e}\right)$. Mol Ecol Res 13, 243-253. 
22. Waples, R.S. and Do, C. (2010) Linkage disequilibrium estimates of contemporary $N_{e}$

240 using highly variable genetic markers: a largely untapped resource for applied

241 conservation and evolution. Evol Appl 3, 244-262.

242 23. Watts, P.C. et al. (2007) Compatible genetic and ecological estimates of dispersal

243 rates in insect (Coenagrion mercuriale: Odonata: Zygoptera) populations: analysis of

244 'neighbourhood size' using a more precise estimator. Mol. Ecol. 16 (4), 737-51.

245 24. Barclay, H.J. and Hargrove, J.W. (2005) Probability models to facilitate a declaration of 246 pest-free status, with special reference to tsetse (Diptera: Glossinidae). Bull Entomol Res $24795(1), 1-11$.

248 25. Fox, J. (2005) The R commander: a basic statistics graphical user interface to R. J Stat 249 Software $14(9), 1-42$.

250 26. Fox, J. (2007) Extending the R commander by "plug in" packages. R News 7 (3), 4625152.

252 27. R-Core-Team, R: A Language and Environment for Statistical Computing, R 253 Foundation for Statistical Computing, Vienna, Austria, http://www.R-project.org, 2018.

254 28. Baines, C.B. et al. (2014) The interactive effects of competition and predation risk on 255 dispersal in an insect. Biol Let 10 (6), 20140287.

256 29. Ronnås, C. et al. (2011) Limited emigration from an outbreak of a forest pest insect. 257 Mol Ecol 20 (22), 4606-4617.

258 30. Matthysen, E. (2005) Density-dependent dispersal in birds and mammals. Ecography $25928(3), 403-416$.

260 31. Kamioka, T. and Iwasa, Y. (2017) Evolution of density-dependent wing polymorphism 261 in insects. Evol. Ecol. Res. 18 (3), 335-348.

262 32. Rogers, D.J. and Randolph, S.E. (1984) A review of density-dependent processes in 263 tsetse populations. Insect Science and Its Application 5 (5), 397-402. 
33. Gouteux, J.P. et al. (2001) A density-dependent model with reinvasion far estimating tsetse fly populations (Diptera : Glossinidae) through trapping. Bull Entomol Res 91 (3), 177-183.

34. Hargrove, J.W. (2003) Tsetse eradication: sufficiency, necessity and desirability, DFID Animal Health Programme, eds, Centre for Tropical Veterinary Medicine, University of 269 Edinburgh.

270 35. Peck, S.L. and Bouyer, J. (2012) Mathematical modeling, spatial complexity, and 271 critical decisions in tsetse control. J Econ Entomol 105 (5), 1477-1486.

272 36. Cecilia, H. et al. (2018) Environmental heterogeneity drives tsetse fly population 273 dynamics. bioRxiv, 493650 .

274 37. Séré, M. et al. (2017) Comparison of different genetic distances to test isolation by 275 distance between populations. Heredity 119, 55-63.

276 38. Wright, S. (1965) The interpretation of population structure by F-statistics with special 277 regard to system of mating. Evolution 19, 395-420.

278 39. Weir, B.S. and Cockerham, C.C. (1984) Estimating F-statistics for the analysis of 279 population structure. Evolution 38, 1358-1370.

280 40. Rousset, F. (1997) Genetic differentiation and estimation of gene flow from F-statistics 281 under isolation by distance. Genetics 145 (4), 1219-1228.

282 41. Rousset, F. (2000) Genetic differentiation between individuals. J Evol Biol 13 (1), 5828362.

284 42. Cavalli-Sforza, L.L. and Edwards, A.W.F. (1967) Phylogenetic analysis: model and 285 estimation procedures. Am J Hum Genet 19, 233-257.

286 43. Takezaki, N. and Nei, M. (1996) Genetic distances and reconstruction of phylogenetic 287 trees from microsatellite DNA. Genetics 144 (1), 389-399.

288 44. Balloux, F. (2004) Heterozygote excess in small populations and the heterozygote289 excess effective population size. Evolution 58 (9), 1891-900. 
45. Vitalis, R. and Couvet, D. (2001) ESTIM 1.0: a computer program to infer population 291 parameters from one- and two-locus gene identity probabilities. Mol. Ecol. Notes 1 (4), 292 354-356.

293 46. Wang, J.L. and Whitlock, M.C. (2003) Estimating effective population size and 294 migration rates from genetic samples over space and time. Genetics 163 (1), 429-446. 295 47. Mantel, N. (1967) The detection of disease clustering and a generalized regression 296 approach. Cancer Res 27, 209-220.

297 48. WHO, Control and surveillance of human African trypanosomiasis: report of a WHO 298 expert committee, World Health Organization \& WHO Expert Committee on the Control 299 and Surveillance of Human African Trypanosomiasis, World Health Organization. 300 http://www.who.int/iris/handle/10665/95732 Geneva, Switzerland, 2013.

301 49. Bouyer, J. et al. (2007) Learning influences host choice in tsetse. Biol Let 3 (2), 113302116.

303 50. Vale, G.A. ( 1974) Direct observations on the responses of tsetse flies (Diptera, 304 Glossinidae) to hosts. Bull Entomol Res 64, 589-594.

305 51. Randolph, S.E. et al. (1984) Local variation in the population dynamics of Glossina 306 palpalis palpalis (Robineau-Desvoidy) (Diptera: Glossinidae). II. The effect of insecticidal 307 spray programmes. Bull Entomol Res 74, 425-438.

308 52. Rogers, D.J. et al. (1984) Local variation in the population dynamics of Glossina 309 palpalis palpalis (Robineau-Desvoidy) (Diptera: Glossinidae). I. Natural population 310 regulation. Bull Entomol Res 74, 403-423. 
315 Allee effect: A phenomenon where the individual fitness (survival and/or reproduction) is positively correlated with population size or density.

Alleles: The different states of a locus or a gene (e.g. the three alleles of the ABO system for blood groups).

Bootstrap: A randomization procedure where an item series (e.g. loci) are resampled with replacement (the same item can be resampled several times) until the number of items present in the raw data is reached. At each resampling, a statistic is measured (e.g. genetic distance, see Box 1). The procedure is repeated a great number of times (e.g. 5000), which produces a distribution of possible values for the statistic. Excluding the $2.5 \%$ smallest and the $2.5 \%$ biggest values of the bootstrap distribution provides the $95 \%$ bootstrap confidence interval of the statistic.

Correlation coefficient: A measure of the covariation between two variables.

Effective population density: The ratio of effective population size to the surface area occupied by a given population.

Effective population size: Quantifies the rate at which a population loses its genetic diversity. Indeed, the reciprocal of the effective size $(1 / \mathrm{Ne})$ gives the long-term probability that two randomly sampled genes in the population are replicates (or descend) from a single gene in the parental generation. It can be roughly defined as the number of adults in a population that will leave a genetic signature to the next generation. It is generally smaller than the census size $N_{c}$, except when coalescence is delayed due to a particular system of mating (negative assortative mating) or in very small dioecious populations. In any case, $N_{e}$ and $N_{c}$ must be strongly positively correlated in most, if not all, situations.

Immigration rate: The proportion of individuals in a subpopulation that come from other subpopulations of the total population. 
340 Inbreeding: A concept describing how alleles, individuals or subpopulations can be related. Formally, it corresponds to the probability to randomly draw two identical alleles that are identical by descent, i.e. that come from a common ancestor. down for each unit increase in the abscissa.

Linkage disequilibrium: A measure of the statistical correlation between alleles at two or more loci. If in equilibrium, then the occurrence of alleles at two loci is simply equal to the product of corresponding allele frequencies in the population for the two loci. The main forces influencing linkage disequilibrium are reproductive systems, selection and genetic drift. It can be used to measure effective population size (small populations generate and maintain higher linkage disequilibria than bigger ones).

Locus: A specific segment of the genome, not necessarily a coding sequence (gene).

Neighborhood: The number of individuals connected through migration in an isolation by distance framework.

Parametric test: Statistical tests using population parameters (average and variance). If the constraints to apply such tests (e.g. normality of data, homogeneity of variances) are not met, non-parametric tests must be applied instead (e.g. rank tests or Mantel tests).

Population genetics: The study of the distribution of genetic variation in space and time and its evolution with random genetic drift, selection, mutation, migration etc.

Regression: A mathematical model explaining the relationship between a response variable and one (or several) explanatory variable(s).

Riparian: A riparian system describes the interface between land and a river or stream.

Slope: The parameter of a regression that describes how many units ordinates go up or 


\section{Box 1: Measuring genetic distances}

Several genetic distances exist that are used in isolation by distance procedures (see [37] for a more complete overview). The most popular, Wright's FST $_{\text {[38] }}$ is not a real genetic distance but a measure of the effect of subdivision on inbreeding. The parametric definition of this parameter is (e.g. [8]):

$F_{\mathrm{ST}}=\frac{Q_{\mathrm{S}}-Q_{\mathrm{T}}}{1-Q_{\mathrm{T}}}$

where $Q s$ is the probability of identity between two alleles from two individuals of the same subpopulation and $Q_{\mathrm{T}}$ is the probability of identity between two alleles from two subpopulations of the total population. Its value varies between 0 (no subdivision) and 1 (all subsamples fixed for one or the other allele present, i.e. absolute subdivision). It is mainly influenced by subpopulation sizes and immigration rate (or dispersal). This parameter is estimated with Weir and Cockerham's unbiased estimator $\theta$ [39]. For isolation by distance situations, it has been shown that the use of $F_{S T} R=\theta /(1-\theta)$ is more useful because it is linearly related to geographic distances without losing its relation to other demographic parameters [40]. An equivalent measure between individuals $a_{r}$, and it's unbiased estimator â, was also designed by Rousset [41] as: $a_{r}=\frac{Q_{w}-Q_{r}}{1-Q_{w}}$

where $Q_{w}$ is the probability of identity of two genes within an individual and $Q_{r}$ is the probability of identity of genes at (geographical) distance $r$. Another statistic ê can be used in case of very important neighborhood (see [23] for more details). In other instances it may be more appropriate to use another genetic distance, e.g.

386 Cavalli-Sforza and Edward's chord distance [42]. DCSE is more appropriate for tree 387 topology design [43] and more powerful in some cases of isolation by distance testing [37]: 
389 where $r$ is the number of loci, $j$ the locus name (from 1 to $r$ ), $i$ the allele name (from 1 to $390 \mathrm{mj}), m j$ the number of alleles at locus $j, x_{i j}$ and $y_{i j}$ are the frequencies of allele $i$ at locus $j$ for 391 subpopulations $x$ and $y$, respectively.

392

393 
Box 2: Measuring and testing isolation by distance and parameter inferences

Isolation by distance is measured through a regression of geographic distances

396

397

$416 \delta \approx 2 \times \sqrt{\frac{1}{4 \pi b D_{e}}}$

In 2D, immigrants from neighboring subpopulations at each generation can be

(explanatory variable) $D$ Geo between individuals or subsamples and corresponding genetic distances (response variable) $D$ Genet. Inferences follow particular models of regression, depending on whether the population structure occurs in one dimension (1D) or in two dimensions (2D) [23, 40,41]. 1D structures correspond to shores, ecotones or river courses, as it is the case for Glossina palpalis gambiensis along forest galleries in savannas. 2D structures are more common, or at least more often reported. Three dimension models (dense forests or water columns for aquatic organisms) remain poorly explored. In $1 \mathrm{D}$, the model is $D$ Genet $=a+b \times D_{\mathrm{Geo}}$, where $a$ is the intercept, $b$ the slope of the regression and $D_{\text {Genet }}$ Stands for FST_R (for between subpopulations distances), â or ê (between individuals). $\ln 2 \mathrm{D}$, the model is $D_{\mathrm{Genet}}=a+b \times \ln \left(D_{\mathrm{Geo}}\right)$, where $\ln \left(D_{\mathrm{Geo}}\right)$ is the natural logarithm of $D_{\text {Geo. }}$. The slope $b$ is linked to the effective population density $D_{e}$ and the average squared axial parent-offspring distance $\overline{\sigma^{2}}$ with a neighborhood estimated as $N b=4 D_{e} \times \overline{\sigma^{2}}=1 / b$ in $1 \mathrm{D}$ and $N b=4 \pi D_{e} \times \overline{\sigma^{2}}=1 / b$ in $2 \mathrm{D}[40]$.

The average surface $(S)$ occupied by a subpopulation can be computed as the surface area occupied by the different traps used in a given survey site. If only one trap is available per site, the distance between the two closest sites $\left(D_{\min }\right)$ can be taken as the raw proxy of the distance between the centers of two neighboring subpopulations and hence as their diameter: $S=\pi \times\left(D_{\min } / 2\right)^{2}$. If the average effective population size $N_{e}$ is computed with appropriate algorithms ( e.g. see $[20,44-46]$ ), then $D_{e}=N_{e} / S$, and a rough proxy of parent-offspring average distance (dispersal) can be computed as [37]: estimated as $N_{e} m=1 / 2 \pi b[40]$. 
$420 N b<10000$ in $1 \mathrm{D}$ or when $N b<50$ in $2 \mathrm{D}[23]$.

$421 \quad$ Significance testing cannot be undertaken with a parametric test since distance 422 measures are autocorrelated (paired comparisons). The significance of the slope can be 423 tested by a bootstrap over loci based $95 \%$ confidence interval $(95 \% \mathrm{Cl})$. If 0 is not included 424 in $95 \% \mathrm{Cl}$, then the slope is significantly above 0 . Otherwise, a correlation coefficient 425 (e.g. Pearson) is computed between the two distance matrices and cells of one of those 426 permuted a great number of times (Mantel test [47]). The $p$-value of the test is the 427 proportion of time the randomized correlation was as big as or bigger than the observed 428 one. If the $95 \% \mathrm{Cl}$ is not above 0 , the Mantel test may be more powerful if the genetic 429 distance used is $D_{\text {CSE, }}$ at least for highly variable markers like microsatellite loci [37]. 430 


\section{Box 3: Tsetse fly atypical reproduction and density dependence}

Female tsetse flies do not lay eggs but larviposit a single mature larva (3rd instar, L3) in humid soil one at a time. The larva develops feeding from the uterine glands of the mother (adenotrophic viviparity). After larviposition, the larva quickly burrows into the soil surface for pupation. It was shown in G. morsitans that a larviposition pheromone is deposited to attract other females to the same site, leading to a strong aggregation of pupae [48]. The adult emerges $20-80$ days later. Thus, in nature, each female produces no more than $3-5$ offspring during its total life. Lifespan is around 3 months for females, 2 months for males. As a result, the intrinsic rate of tsetse population growth is theoretically low. Both females and males feed on vertebrate blood and are therefore both vectors. Learning capacities of tsetse flies may increase their hunting efficiency with age and encourage those returning to their first host [49].

Evidence for negative density dependence in tsetse flies has been reported in several studies. Interactions between flies and the irritation of the host animal are responsible for a decreased proportion of fed $G$. morsitans morsitans as the numbers of tsetse flies arriving increase, and frequently disturbed spots might encourage tsetse flies to leave without feeding, simultaneously increasing dispersal and associated mortality and decreasing local density $[32,50]$. Host irritation and tsetse learning are important parameters driving the survival and dispersal of tsetse flies.

In dense tsetse populations, the populations are self-sustained and are considerably reduced after insecticidal spray, but are recolonized from neighboring sites, which takes several months to complete [51]. Alternatively, low-density sites are naturally sustained by immigrants from neighboring sites and insecticidal treatments do not have as much impact on the total population [32, 51, 52]. 
457 Figure 1: Strong negative density-dependent dispersal in tsetse fly populations. The graph shows the relationship between effective population densities $D_{e}$ (individuals per $\mathrm{km})$ and dispersal $(\delta$, in $\mathrm{km})(\mathrm{a})$ or between census population densities (densities of captured flies) (b) across different two-dimensional isolation by distance studies of different tsetse fly species in different African countries. The straight line corresponds to the power regression indicated in the graph with its determination coefficient $R^{2}$ and corresponding Spearman's coefficient $(\rho)$ and associated $p$-value. Dashes indicate bootstrap over loci $95 \%$ confidence intervals. The same symbols indicate the same species. Scales were log-transformed for both 466 axes. 
(a)

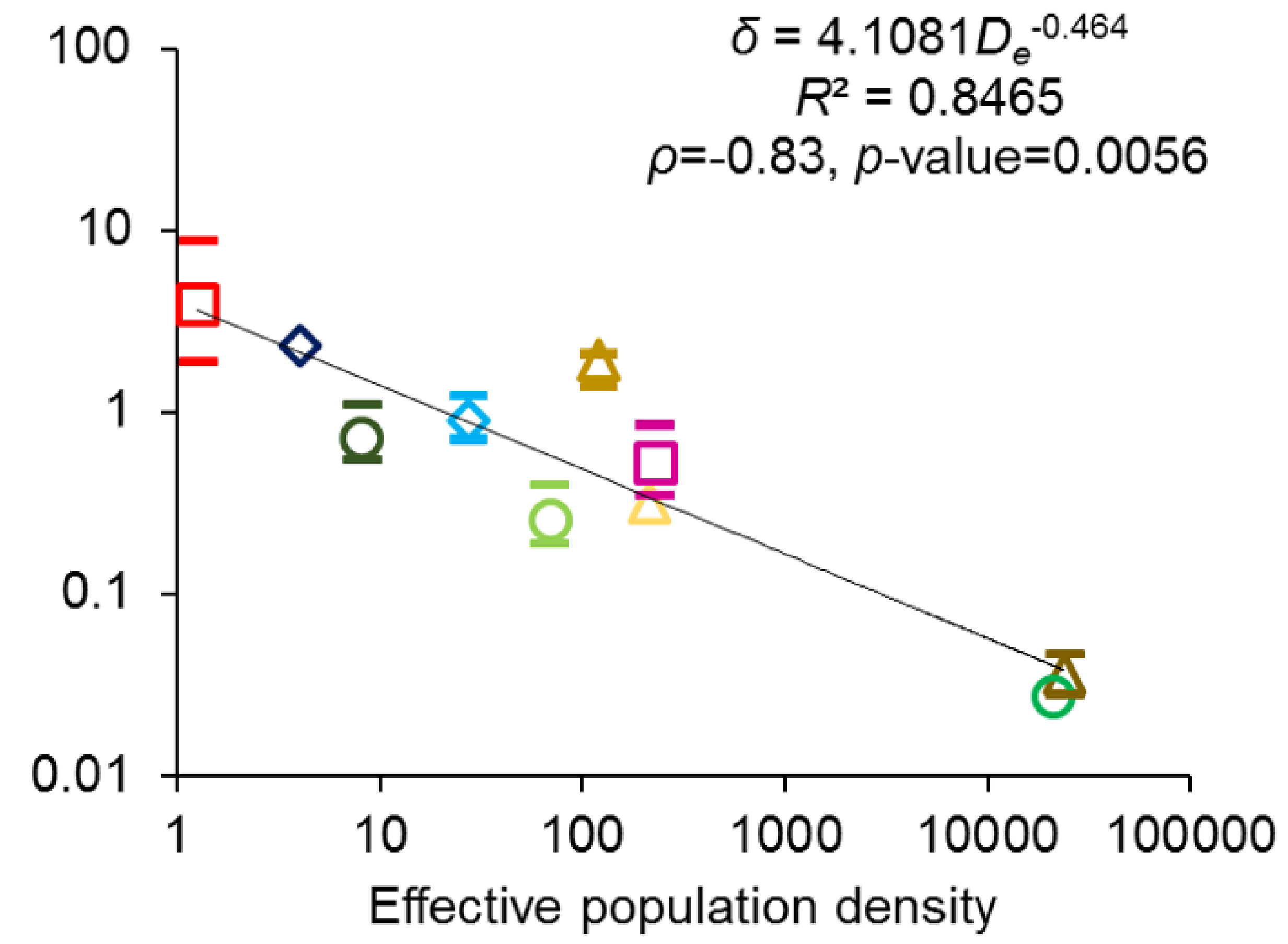

(b)

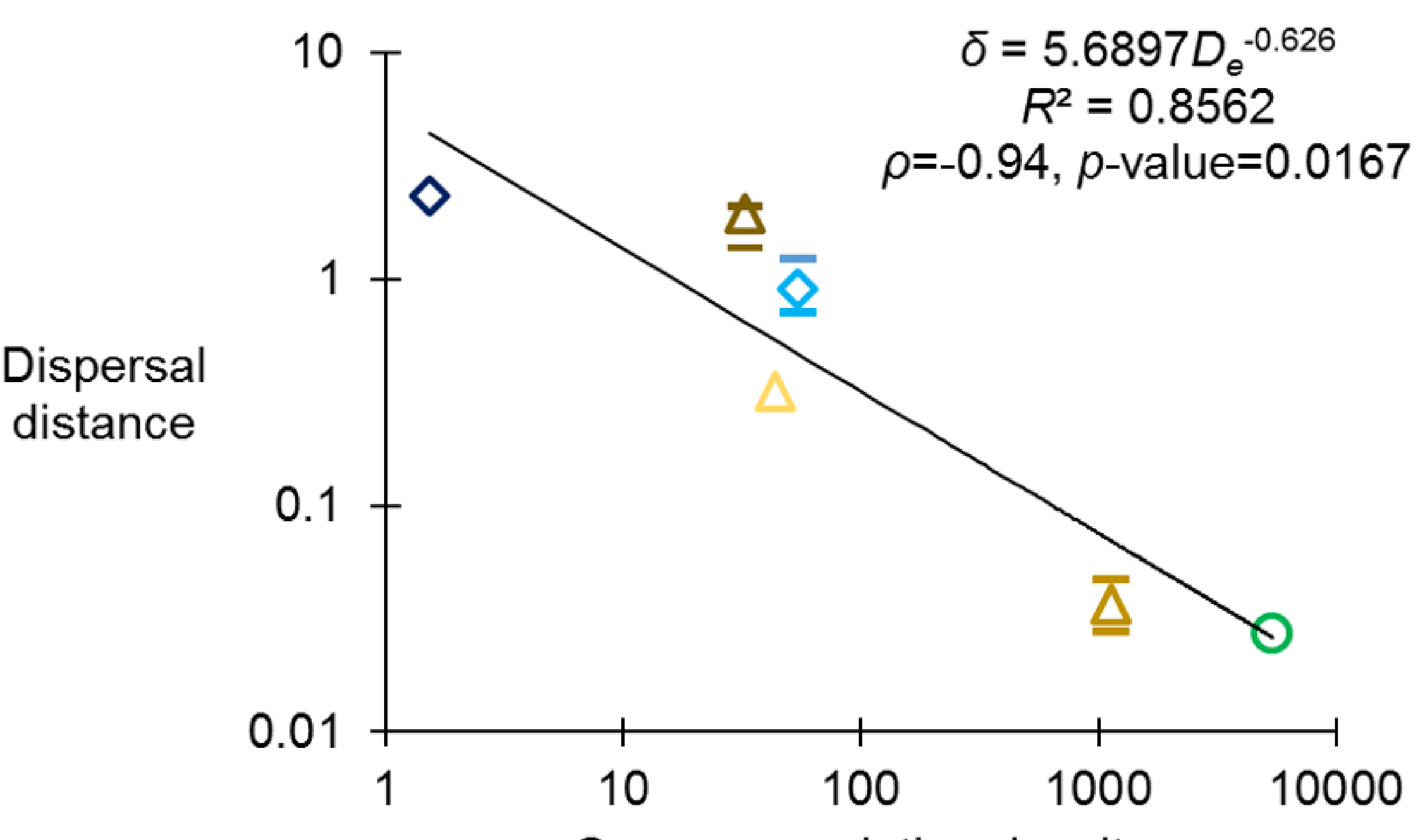

$\square$ Glossina pallidipes from Tanzania

$\square$ Glossina pallidipes from Kenya

$\diamond$ Glossina tachinoides from Burkina Faso

$\diamond$ Glossina tachinoides from Ghana

Glossina fuscipes fuscipes from Uganda 2012

Glossina fuscipes fuscipes from Tanzania and Kenya

Glossina fuscipes fuscipes from Uganda 2017

$\triangle$ Glossina palpalis palpalis from Cameroon 2012

$\triangle$ Glossina palpalis gambiensis from Burkina Faso

$\triangle$ Glossina palpalis palpalis from Cameroon 2015 